\title{
Centralized normalization of harmonic voltages by the third-order passive filter
}

\author{
L.I.Kovernikova \\ The Siberia Branch of the Russian Academy of Sciences \\ Energy Systems Institute \\ 130, Lermontov Str., 664033 Irkutsk (Russia) \\ Phone: +7 3952 426495, fax: +7 3952 426796, e-mail: kovernikova@isem.sei.irk.ru
}

\begin{abstract}
The values of harmonic voltages at network nodes should meet the standard limits. Harmonic voltages can be decreased to admissible values on a centralized basis. The paper addresses the problem of centralized decrease of harmonic voltages in the high voltage networks with distributed nonlinear loads using third-order passive filters. Mathematical expressions to determine parameters of the third-order passive filter are given. The parameters of the selected filters for 220 $\mathrm{kV}$ network supplying traction substations with power and also the estimates of the efficiency of work of these filters for different network configurations are presented as an example. The efficiency of the C-type and third-order filters are compared.
\end{abstract}

\section{Key words}

Harmonic measurement, harmonic distortion, harmonic limits, centralized normalization, filters design.

\section{Introduction}

The problem of harmonic voltage normalization on a centralized basis is considered in [1-3] where the authors suggest applying first-order passive filters for centralized decrease of harmonic voltages in the $12-23 \mathrm{kV}$ distribution networks with a large number of nonlinear loads of small capacity. These filters are widely used. Their parameters are easily calculated.

The present paper addresses the problem of centralized harmonic voltage decrease in the $220 \mathrm{kV}$ network. This network supplies electricity to the traction loads from the $27.5 \mathrm{kV}$ winding of $40 \mathrm{MVA}$ three-phase three-winding transformers. The traction substations are located at a distance of $40-60 \mathrm{~km}$ from one another. Therefore nonlinear loads are numerous and distributed along hundreds of kilometers of $220 \mathrm{kV}$ supply network. This problem has already been considered in [4] where the author shows the possibility to use C-type filters for centralized normalization of harmonic voltages in the network with distributed nonlinear load.

This paper also considers the possibility to use a thirdorder filter for this purpose. The authors in [5] point out that the C-type filter is more sensitive to changes in frequency and variations in parameters of elements, than the third-order filter. It is also noted in [5] that both filters have a great advantage - low losses at the fundamental frequency. Calculation of the third-order filter parameters poses difficulty. In [6] expressions for calculation of the filter parameters are derived for the condition of equal capacitance of two capacitors used in the filters, i.e. $\boldsymbol{C}_{1}=\boldsymbol{C}_{2}$. In [5] the authors indicate that in the third-order filter there should be a relation $\boldsymbol{C}_{1} \gg \boldsymbol{C}_{2}$. In this paper the expressions for calculation of the filter parameters if $\boldsymbol{C}_{1} \neq \boldsymbol{C}_{2}$ are obtained and possible relations between $\boldsymbol{C}_{1}$ and $\boldsymbol{C}_{2}$ are studied. The possibility of using the third-order filter to normalize harmonic voltages on a centralized basis is shown and its comparison with the C-type filter is made.

\section{Determination of filter parameters}

The scheme of the third-order filter is given in Fig.1. It is necessary to determine the parameters $\boldsymbol{X}_{\boldsymbol{C} 1}, \boldsymbol{X}_{\boldsymbol{C} 2}, \boldsymbol{R}$, $\boldsymbol{X}_{\boldsymbol{L}}$ at the fundamental frequency. The value of $\boldsymbol{R}$ is assumed to be invariable with change in the frequency. Resistance of reactor is not taken into account. Reactance $\boldsymbol{X}_{\boldsymbol{C} 1}$ is calculated by the set values of reactive 
power $\boldsymbol{Q}_{\boldsymbol{C} 1}$ and voltage $\boldsymbol{U}$ at the fundamental frequency as

$$
\begin{gathered}
\boldsymbol{X}_{\boldsymbol{C} 1}=\sqrt{3} \boldsymbol{U}^{2} / \boldsymbol{Q}_{\boldsymbol{C} 1} . \\
\frac{\mathrm{Xc1}}{\square}=\mathrm{Xc2} \\
\frac{\mathrm{R}}{\mathrm{R}}
\end{gathered}
$$

Fig. 1. Schematic diagram of the third-order filter.

The remaining filter parameters should meet the following conditions:

1) resistance of filter at the $\boldsymbol{n}$-th harmonic equals $\boldsymbol{R}_{\boldsymbol{F}}$,

2) reactance of filter at the $\boldsymbol{n}$-th harmonic equals zero,

3) $\boldsymbol{X}_{\boldsymbol{C} 2}=\boldsymbol{m} \boldsymbol{X}_{\boldsymbol{C} 1}$,

where $\boldsymbol{m}$ - a coefficient taking into account relations between $\boldsymbol{X}_{\boldsymbol{C} 1}$ and $\boldsymbol{X}_{\boldsymbol{C} 2}$.

To reduce harmonic voltages at the network nodes to the $(0.5-0.75)$ of the $\boldsymbol{K}_{U(n) N}$ values at the tuned harmonic the filter should have a certain value of resistance $\boldsymbol{R}_{\boldsymbol{F}}$. $\boldsymbol{K}_{\boldsymbol{U}(\boldsymbol{n}) \boldsymbol{N}}$ is a norm for the index $\boldsymbol{K}_{\boldsymbol{U}(\boldsymbol{n})}$ [7]. The value of $\boldsymbol{R}_{\boldsymbol{F}}$ is calculated by the expression given in [4]

$$
\boldsymbol{R}_{F}=\frac{\boldsymbol{K}_{U(n) F}}{\left(K_{U(n)}-K_{U(n) F}\right) \sqrt{g_{n s}{ }^{2}+b_{n s}^{2}}}
$$

где $\boldsymbol{K}_{\boldsymbol{U}(\boldsymbol{n})}, \boldsymbol{K}_{\boldsymbol{U}(\boldsymbol{n}) \boldsymbol{F}}$ - values of indices at the node without the filter and with it,

$\boldsymbol{g}_{\boldsymbol{n} \boldsymbol{s}}, \boldsymbol{b}_{\boldsymbol{n} \boldsymbol{s}}$ - conductance and susceptance of network at the filter connection node.

Impedance of the third-order filter at the $\boldsymbol{n}$-th harmonic can be written as

$$
Z_{n}=\frac{j X_{L n}\left(R-j X_{C 2 n}\right)}{R+j\left(X_{L n}-X_{C 2 n}\right)}-j X_{C 1 n} .
$$

Transform $\boldsymbol{Z}_{\boldsymbol{n}}$, by separating real and imaginary parts

$$
\begin{gathered}
Z_{n}=\frac{R X_{L n}^{2}}{R^{2}+X_{L C 2 n}^{2}}+ \\
+j \frac{R^{2} X_{L C 1 n}-X_{L n} X_{C 2 n} X_{L C 2 n}-X_{C 1 n} X_{L C 2 n}^{2}}{R^{2}+X_{L C 2 n}^{2}},
\end{gathered}
$$

where $\boldsymbol{X}_{\boldsymbol{L C 1 n}}=\boldsymbol{X}_{\boldsymbol{L n}}-\boldsymbol{X}_{\boldsymbol{C} 1 \boldsymbol{n}}$,

$$
X_{L C 2 n}=X_{L n}-X_{C 2 n}
$$

According to conditions 1) and 2) imaginary part $\boldsymbol{Z}_{\boldsymbol{n}}$ at the $\boldsymbol{n}$-th harmonic is equal to zero, real - to $\boldsymbol{R}_{\boldsymbol{F}}$. After the transformations real and imaginary part $\boldsymbol{Z}_{\boldsymbol{n}}$ can be represented as a system of two equations

$$
\begin{aligned}
& R X_{L n}^{2}-R_{f} R^{2}-R_{f} X_{L C 2 n}^{2}=0 \\
& R^{2} X_{L C 1 n}-X_{L n} X_{C 2 n} X_{L C 2 n}-X_{C 1 n} X_{L C 2 n}^{2}=0 .
\end{aligned}
$$

Having solved the system of equations, considering that $\boldsymbol{X}_{\boldsymbol{C} 2}=\boldsymbol{m} \boldsymbol{X}_{\boldsymbol{C} 1}$, we obtain the expressions for $\boldsymbol{X}_{\boldsymbol{L n}}$ and $\boldsymbol{R}$

$$
\begin{gathered}
\boldsymbol{X}_{\boldsymbol{L n}}=\left(-\boldsymbol{B}+\sqrt{\boldsymbol{B}^{2}-4 \boldsymbol{A C}}\right) /(2 \boldsymbol{A}), \\
\boldsymbol{R}=\boldsymbol{R}_{\boldsymbol{F}}\left(\boldsymbol{X}_{\boldsymbol{L} \boldsymbol{n}}-\boldsymbol{m} \boldsymbol{X}_{\boldsymbol{C} 1 \boldsymbol{n}}\right) / \boldsymbol{X}_{\boldsymbol{L C} 1 \boldsymbol{n}} \\
\text { where } \boldsymbol{A}=-\boldsymbol{X}_{\boldsymbol{C} 1 \boldsymbol{n}}(1+\boldsymbol{m}) \\
\boldsymbol{B}=\boldsymbol{R}_{\boldsymbol{F}}^{2}+\boldsymbol{X}_{\boldsymbol{C} 1 \boldsymbol{n}}^{2}(1+2 \boldsymbol{m}) \\
\boldsymbol{C}=-\boldsymbol{R}_{\boldsymbol{F}}^{2} \boldsymbol{m} \boldsymbol{X}_{\boldsymbol{C} 1 \boldsymbol{n}}\left(1+\boldsymbol{X}_{\boldsymbol{C} 1 \boldsymbol{n}}^{2}\right)
\end{gathered}
$$

The value of $\boldsymbol{m}$ is unknown in the obtained expressions. In order to determine the admissible values of $\boldsymbol{m}$ we use the conditions that $\boldsymbol{C}_{1}>>\boldsymbol{C}_{2}$ and the expression under the root sign in (4) should be positive, i.e.

$$
\boldsymbol{B}^{2}-4 \boldsymbol{A C}>0 \text {. }
$$

Solving the inequality we obtain the value of $\boldsymbol{m}$ lying in the interval

$$
1<\boldsymbol{m}<\frac{\boldsymbol{R}_{\boldsymbol{F}}^{2} \boldsymbol{n}^{2}+\boldsymbol{X}_{\boldsymbol{C} 1}^{2}}{2 \boldsymbol{n} \boldsymbol{R}_{\boldsymbol{F}} \boldsymbol{X}_{\boldsymbol{C} 1}} .
$$

(9)

Expression (9) shows that the relation between $\boldsymbol{X}_{\boldsymbol{C} 1}$ and $\boldsymbol{X}_{\boldsymbol{C} 2}$ is determined by the values $\boldsymbol{X}_{\boldsymbol{C} 1}$ and $\boldsymbol{R}_{\boldsymbol{F}}$ at the harmonic for which the filter is chosen. Thus, the parameters of the third-order filter on the basis of (2), (4)-(9) can be calculated by the expressions:

$$
\begin{aligned}
& \boldsymbol{X}_{\boldsymbol{C} 1}=\sqrt{3} \boldsymbol{U}^{2} / \boldsymbol{Q}_{\boldsymbol{C} 1}, \\
& \boldsymbol{X}_{\boldsymbol{C} 2}=\boldsymbol{m} \boldsymbol{X}_{\boldsymbol{C} 1}, \\
& \boldsymbol{X}_{\boldsymbol{L}}=\left(-\boldsymbol{B}+\sqrt{\boldsymbol{B}^{2}-4 \boldsymbol{A C}}\right) /(2 \boldsymbol{A n}), \\
& \boldsymbol{R}=\boldsymbol{R}_{\boldsymbol{F}}\left(\boldsymbol{X}_{\boldsymbol{L}} \boldsymbol{n}^{2}-\boldsymbol{m} \boldsymbol{X}_{\boldsymbol{C} 1}\right) /\left(\boldsymbol{X}_{\boldsymbol{L}} \boldsymbol{n}^{2}-\boldsymbol{X}_{\boldsymbol{C} 1}\right) .
\end{aligned}
$$

Below the obtained expressions are used to choose the filters for the $220 \mathrm{kV}$ network which supplies power to the traction load.

\section{Example of filter selection}

\section{A. Characteristics of harmonic distortions in the considered network}

The $220 \mathrm{kV}$ network is $900 \mathrm{~km}$ long. It supplies power to 23 traction substations. A more detailed description of the network is given in [4]. The indices $\boldsymbol{K}_{\boldsymbol{U}(\boldsymbol{n})}$ (the $\boldsymbol{n}$-th harmonic factor) and $\boldsymbol{K}_{\boldsymbol{U}}$ (the total harmonic distortion) were measured at seven nodes of the network. The measurement results are presented in Table 1. The same Table shows normally admissible values of $\boldsymbol{K}_{\boldsymbol{U}(\boldsymbol{n})}$ and $\boldsymbol{K}_{\boldsymbol{U}}$ determined in [7]. The measured values of $\boldsymbol{K}_{\boldsymbol{U}(\boldsymbol{n})}$ and $\boldsymbol{K}_{\boldsymbol{U}}$ that exceed the normally admissible values are given in bold type. The admissible values of $\boldsymbol{K}_{\boldsymbol{U}(\boldsymbol{n})}$ and $\boldsymbol{K}_{\boldsymbol{U}}$ are exceeded at four substations. 
TABLE 1. - Measured $\boldsymbol{K}_{\boldsymbol{U}(\boldsymbol{n})}$ and $\boldsymbol{K}_{\boldsymbol{U}}$

\begin{tabular}{|c|c|c|c|c|}
\hline Substation & $\begin{array}{c}\boldsymbol{K}_{\boldsymbol{U}} \\
(\%)\end{array}$ & $\begin{array}{c}\boldsymbol{K}_{\boldsymbol{U}(3)} \\
(\%)\end{array}$ & $\begin{array}{c}\boldsymbol{K}_{\boldsymbol{U}(5)} \\
(\%)\end{array}$ & $\begin{array}{c}\boldsymbol{K}_{\boldsymbol{U}(7)} \\
(\%)\end{array}$ \\
\hline S1 & 1.99 & 0.76 & 1.34 & 0.92 \\
\hline S2 & 1.92 & 0.84 & 1.13 & 0.81 \\
\hline S3 & 1.91 & 0.89 & 1.02 & 0.70 \\
\hline S6 & $\mathbf{2 . 5 4}$ & 1.00 & $\mathbf{2 . 3 2}$ & 0.76 \\
\hline S12 & $\mathbf{2 . 0 1}$ & $\mathbf{1 . 7 1}$ & 1.22 & 0.69 \\
\hline S13 & $\mathbf{2 . 8 6}$ & $\mathbf{2 . 0 0}$ & $\mathbf{2 . 0 7}$ & 0.82 \\
\hline S14 & $\mathbf{3 . 0 9}$ & $\mathbf{2 . 0 6}$ & $\mathbf{2 . 2 7}$ & 0.96 \\
\hline Norms & 2.0 & 1.5 & 1.5 & 1.0 \\
\hline
\end{tabular}

The values of $\boldsymbol{K}_{\boldsymbol{U}(\boldsymbol{n})}$ and $\boldsymbol{K}_{\boldsymbol{U}}$ at the other nodes of the network were calculated by the software HARMONICS and are presented in Fig.2. The admissible values of $\boldsymbol{K}_{\boldsymbol{U}(3)}$ are exceeded at eight nodes, $\boldsymbol{K}_{\boldsymbol{U}(5)}$ - at twelve, $\boldsymbol{K}_{\boldsymbol{U}(7)}$ - at four, $\boldsymbol{K}_{\boldsymbol{U}}$ - at twelve nodes and should be reduced with the help of filters.

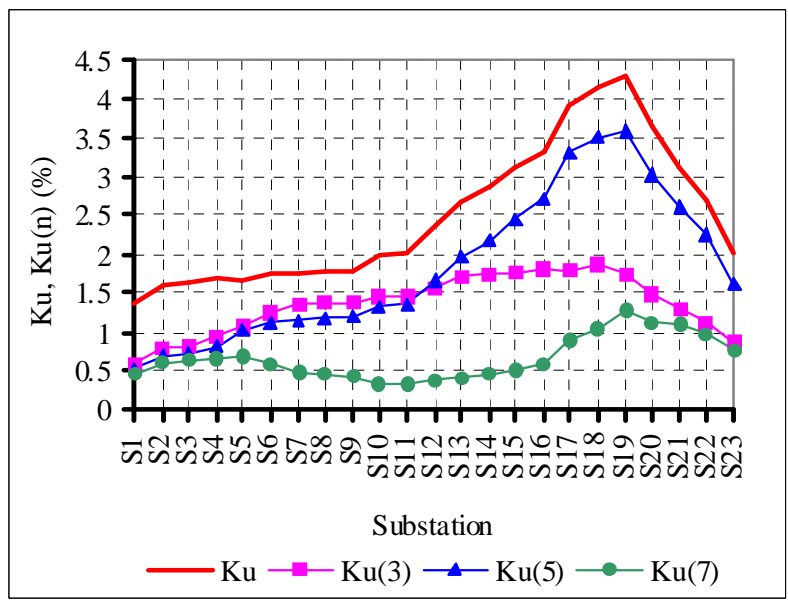

Fig. 2. $\boldsymbol{K}_{\boldsymbol{U}(\boldsymbol{n})}$ and $\boldsymbol{K}_{\boldsymbol{U}}$ at the connection nodes of substations.

Generally, the filters are chosen first for the lowest harmonic. In the given case this is 3rd harmonic. For the next harmonics the filters are chosen on the basis of those previously chosen. Below the choice of the filter for 5th harmonic is presented as an example.

\section{B. Choice of node for installation of filters}

Normal operating conditions were assumed as calculated conditions to choose filters for the considered network. The measurements of $\boldsymbol{K}_{\boldsymbol{U}(\boldsymbol{n})}$ and $\boldsymbol{K}_{\boldsymbol{U}}$ were carried out for these conditions. The first task when choosing filters is to identify the node for their installation. The choice of node intended for filter installation is made with the help of a "test filter" [4]. In Fig.3 the name of each cirve indicates the node for installation of the "test filter" and the curves show the impact of filter on the value of $\boldsymbol{K}_{\boldsymbol{U}(5)}$. For example the filter placed at node S11 can increase the values of $\boldsymbol{K}_{\boldsymbol{U}(5)}$ as compared to the network operation without the filter. The most suitable node for the 5th harmonic filter is node S17. The filter decreases the value of $\boldsymbol{K}_{\boldsymbol{U}(5)}$ practically at all nodes of the network. The curve of $\boldsymbol{K}_{\boldsymbol{U}(5)}$ changes along the entire trajectory of nodes without dramatic changes in the value of $\boldsymbol{K}_{\boldsymbol{U}(5)}$. For the 3rd and 7th harmonics node S17 has also turned out to be the most suitable.

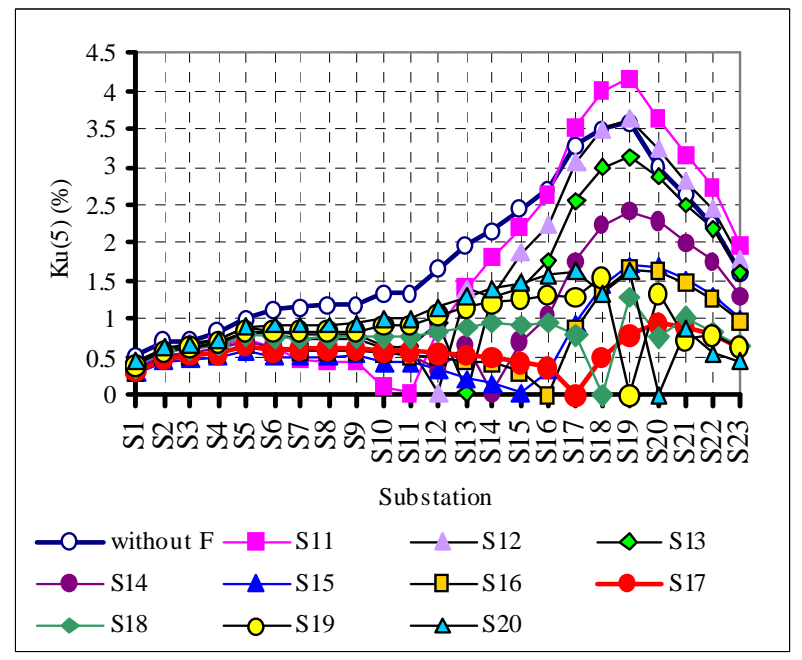

Fig.3. $\boldsymbol{K}_{\boldsymbol{U}(5)}$ at nodes of the network with test filter.

\section{Determination of parameters of the harmonic filters}

The filter parameters of 5 th harmonic will be selected so that the values of $\boldsymbol{K}_{\boldsymbol{U}(5) \boldsymbol{F}}$ at the network nodes are 0.7$1.2 \%$ and the value of the reactive power of the fundamental harmonic is 4-6 Mvar. The choice should be made of such filter parameters that the power losses in the filter were the lowest. The below calculations on the selection of filter parameters were performed by the software HARMONICS.

\section{1) Calculation of the filter resistance $\boldsymbol{R}_{\boldsymbol{F}}$. The} values of the filter resistance $\boldsymbol{R}_{\boldsymbol{F}}$ for the desired values of $\boldsymbol{K}_{\boldsymbol{U}(5) \boldsymbol{F}}$ were calculated by expression (1). Fig. 4 presents curves of the resistance $\boldsymbol{R}_{\boldsymbol{F}}$, and also the power losses $\boldsymbol{P}_{(5)}$ for 5th harmonic in the filter at the calculated values of $\boldsymbol{R}_{\boldsymbol{F}}$.

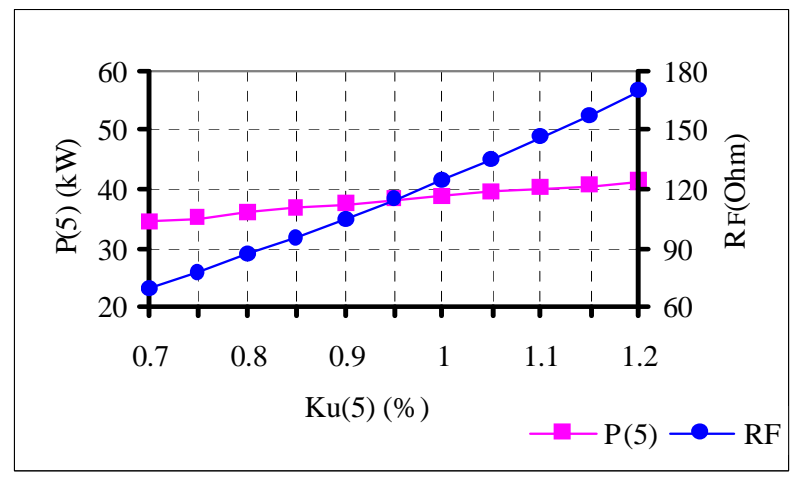

Fig. 4. $\boldsymbol{R}_{\boldsymbol{F}}$ and $\boldsymbol{P}_{(5)}$ with change of $\boldsymbol{K}_{\boldsymbol{U}(5) \boldsymbol{F}}$. 
As a result of analysis it was assumed to calculate the filter parameters for $\boldsymbol{K}_{\boldsymbol{U}(5) \boldsymbol{F}}=0.7 \%$, which is achieved at $\boldsymbol{R}_{\boldsymbol{F}}=69.2 \mathrm{Ohm}$ and the power losses of $34.3 \mathrm{~kW}$. The value of $\boldsymbol{Q}_{\boldsymbol{C} 1}$ does not affect $\boldsymbol{R}_{\boldsymbol{F}}$. For the 5th harmonic filter it was assumed that $\boldsymbol{Q}_{\boldsymbol{C} 1}=3$ Mvar.

2) Calculation of the parameter $\boldsymbol{m}$. The range of values of the parameter $\boldsymbol{m}$ was calculated by relation (9). Table 2 presents the minimum and maximum values of $\boldsymbol{m}$ for $\boldsymbol{K}_{\boldsymbol{U}(5) \boldsymbol{F}}$ over the range 0.7-1.2\% and $\boldsymbol{Q}_{\boldsymbol{C} 1}$ over the range 4-6 Mvar. For $\boldsymbol{R}_{\boldsymbol{F}}=69.2 \mathrm{Ohm}$ and $\boldsymbol{Q}_{\boldsymbol{C} 1}=3$ Mvar the values of $\boldsymbol{m}$ must be in the range

$$
2.33<\boldsymbol{m} \leq 20.99 \text {. }
$$

TABLE 2. - Values $\boldsymbol{m}$

\begin{tabular}{|c|c|c|c|c|}
\hline $\boldsymbol{K}_{\boldsymbol{U}(5)}$ & \multicolumn{4}{|c|}{$\boldsymbol{Q}_{\boldsymbol{C} 1}$ (Mvar) } \\
\cline { 2 - 5 }$(\%)$ & 3 & 4 & 5 & 6 \\
\hline 0.7 & 2.33 & 1.75 & 1.4 & 1.17 \\
\cline { 2 - 5 } & 20.99 & 15.75 & 12.65 & 10.51 \\
\hline 0.8 & 1.87 & 1.40 & 1.12 & 0.94 \\
\cline { 2 - 5 } & 16.85 & 12.64 & 10.12 & 8.44 \\
\hline 0.9 & 1.54 & 1.16 & 0.93 & 0.77 \\
\cline { 2 - 5 } & 13.89 & 10.43 & 8.35 & 6.97 \\
\hline \multirow{2}{*}{1.0} & 1.30 & 0.97 & 0.78 & 0.65 \\
\cline { 2 - 5 } & 11.68 & 8.77 & 7.03 & 5.87 \\
\hline \multirow{2}{*}{1.1} & 1.11 & 0.83 & 0.67 & 0.56 \\
\cline { 2 - 5 } & 9.96 & 7.48 & 6.00 & 5.01 \\
\hline 1.2 & 0.95 & 0.72 & 0.57 & 0.48 \\
\cline { 2 - 5 } & 8.58 & 6.45 & 5.17 & 4.32 \\
\hline
\end{tabular}

3) Calculation of the filter parameters. The filter parameters were calculated by applying 10 values of $\boldsymbol{m}$ with a step of $\Delta \boldsymbol{m}=2.33$. The parameters and the input resistance and reactance of the filter for harmonics from 1 to 25 that are presented in Figs. 5 and 6 were calculated for these values based on expressions (10)-(13).

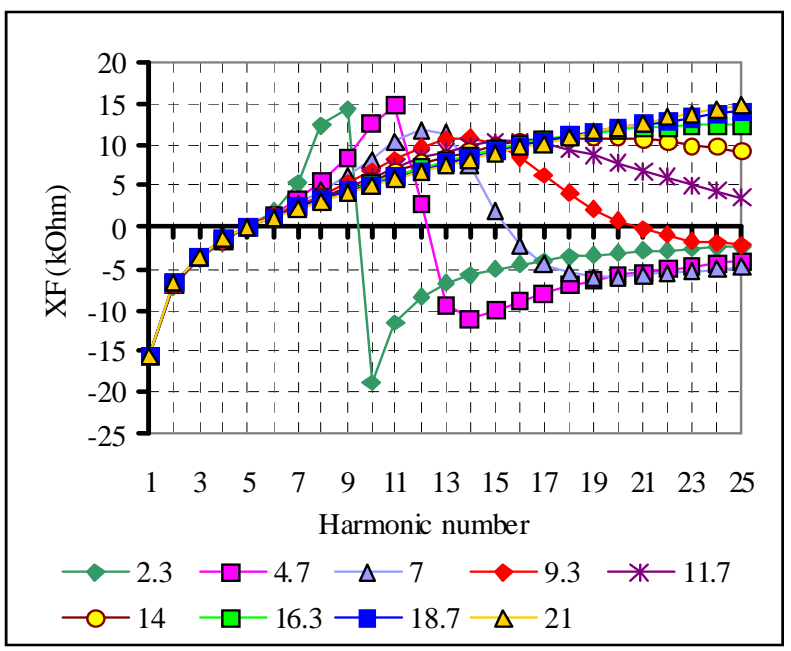

Fig. 5. Input filters reactance for different values of $\boldsymbol{m}$.
The curves in Fig. 5 show that for the harmonics below the 5 th one the filter reactance is capacitive. For the harmonics above the 5th one for $\boldsymbol{m} \leq 9.3$ the filter reactance is also capacitive for some harmonics, which can create resonance circuits with the network. The input filter resistance of the harmonics below the 5th one is low. For the harmonics above the 5 th one the resistances for some $\boldsymbol{m}$ have high values, which can cause large power losses in the filter, if there are currents of these harmonics in the network. Analysis of the curves shows that $\boldsymbol{m}$ equal to 20.99 is the most suitable for selecting the filter. The parameters corresponding to this value of $\boldsymbol{m}$ were selected for the 5th harmonic filter.

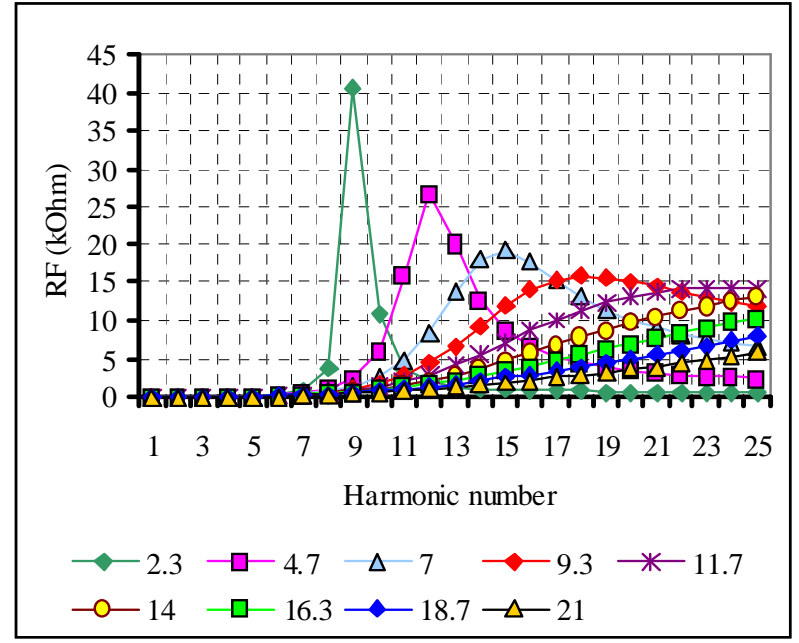

Fig. 6. Input filters resistance for different values of $\boldsymbol{m}$.

The filter parametrs of the 3rd and 7th harmonics were determined in a similar way. The filter parameters are presented in Table 3.

TABLE 3. - Parameters of filters

\begin{tabular}{|l|c|c|c|}
\hline \multirow{2}{*}{ Parameter } & \multicolumn{3}{|c|}{ Harmonic } \\
\cline { 2 - 4 } & 3 & 5 & 7 \\
\hline $\boldsymbol{Q}_{\boldsymbol{C} 1}(\mathrm{Mvar})$ & 4. & 3. & 3. \\
\hline $\boldsymbol{X}_{\boldsymbol{C} 1}(\mathrm{Ohm})$ & 12100.0 & 16133.3 & 16133.3 \\
\hline $\boldsymbol{X}_{\boldsymbol{C} 2}(\mathrm{Ohm})$ & 191180.7 & 338639.1 & 111166.0 \\
\hline $\boldsymbol{X}_{\boldsymbol{L}}(\mathrm{Ohm})$ & 1287.0 & 624.3 & 299.3 \\
\hline $\boldsymbol{R}(\mathrm{Ohm})$ & 39940.7 & 42446.9 & 9954.0 \\
\hline
\end{tabular}

The curves of $\boldsymbol{K}_{\boldsymbol{U}(3)}, \boldsymbol{K}_{\boldsymbol{U}(5)}, \boldsymbol{K}_{\boldsymbol{U}(7)}$ at the network nodes with filters are shown in Fig. 7. At all the network nodes the filters decrease the values of indices to the desired values.

\section{Efficiency estimation of the filters}

The efficiency of filter operation was tested in 18 operating conditions of the supply main. The desired values of $\boldsymbol{K}_{\boldsymbol{U}(7)}$ were exceeded only in one operating condition at two nodes for 7 th harmonic. 


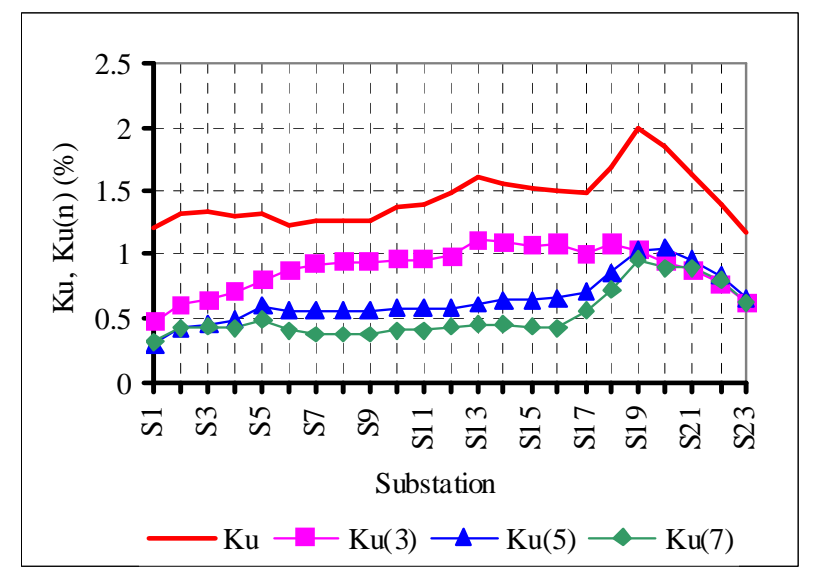

Fig.7. $\boldsymbol{K}_{\boldsymbol{U}}$ и $\boldsymbol{K}_{\boldsymbol{U}(\boldsymbol{n})}$ at the network nodes with filters.

Besides, the impact of the selected filters on operating conditions of harmonics 11, 13, 17, 19, 23 and 25 was analyzed at all the nodes. Fig. 8 shows the curves of $\boldsymbol{K}_{\boldsymbol{U}(11)}$ and $\boldsymbol{K}_{\boldsymbol{U}(13)}$ without filters and with them. It is seen from the Figure that the impact of filters for harmonics 3, 5 and 7 on voltages of harmonics 11 and 13 at all the network nodes is negligible. There is practically no impact of filters for the remaining harmonics.

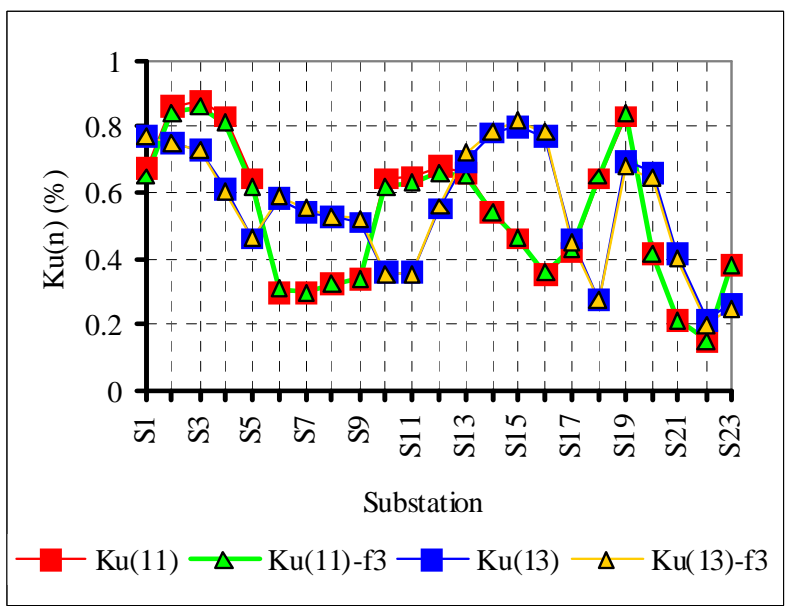

Fig.8. $\boldsymbol{K}_{\boldsymbol{U}(\boldsymbol{n})}$ at the network nodes without filters and with filters.

\section{Comparative estimation of the efficiency of the third-order and C-type filters at centralized normalization of harmonic voltages}

In [4] the centralized normalization of the voltages of harmonics 3, 5 and 7 for the considered network was performed by using the C-type filters. Fig. 9 presents the curves of $\boldsymbol{K}_{\boldsymbol{U}(3)}, \boldsymbol{K}_{\boldsymbol{U}(5)}, \boldsymbol{K}_{\boldsymbol{U}(7)}$ at the network nodes with the third-order and C-type filters. The values of indices at the network nodes with both types of filters differ very slightly.

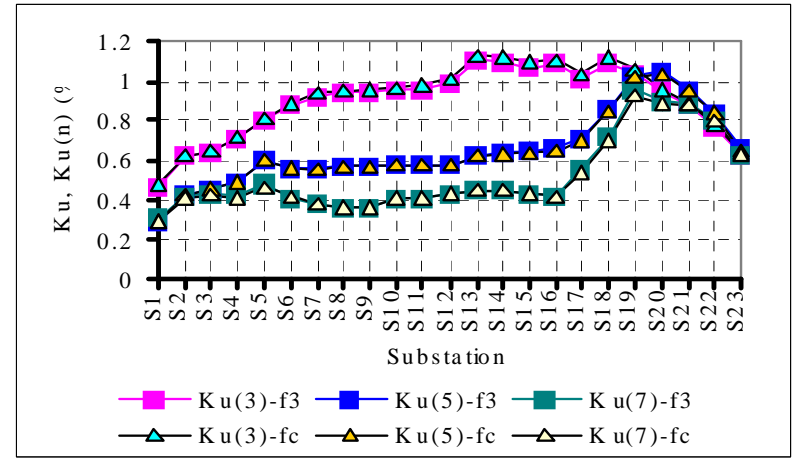

Fig.9. $\boldsymbol{K}_{\boldsymbol{U}(\boldsymbol{n})}$ with the third-order and C-type filters.

\section{Conclusions}

1. The possibility of using the third-order filters for centralized normalization of harmonic voltages is shown.

2. The mathematical expressions for calculation of the third-order filter parameters are derived.

3. The third-order filters for harmonics 3,5 and 7 for the $220 \mathrm{kV}$ network are selected that decrease harmonic voltages in the network about $900 \mathrm{~km}$ long on a centralized basis.

4. Comparative estimation of the efficiency of decreasing voltages of harmonics 3,5 and 7 is made by the thirdorder and C-type filters. It has been revealed that they reduce harmonic voltages virtually equally.

\section{Acknowledgement}

The work was supported by the grant of the Leading Scientific School of the RF SS-4633.2010.8.

\section{References}

[1] Thomas H. Ortmeyer, Takashi Hiyama. Distribution system harmonic filter planning. IEEE Transaction on Power Delivery, vol.11, No. 4, October 1996, pp. 2005-2012.

[2] Kawann C., Emanuel A.E. Passive shunt harmonic filters for low and medium voltage: a cost comparison study. IEEE Transactions on Power Systems, Vol. 11, No. 4, November 1996, pp. 1825-1831.

[3] Gary W. Chang, Hung-Lu Wang, Gen-Sheng Chuang, Shou-Yung Chu. Passive harmonic filter planning in a power system with considering probabilistic constraints. IEEE Transaction on Power Delivery, vol.24, No.1, January 2009, pp. 208-217.

[4] Kovernikova L.I. Centralized normalization of harmonic voltages in the supply network for traction substations. ICREPQ'09, Valencia, 15-17 of April, 2009.

[5] Arrillaga, Jos. Power system harmonics /J. Arrillaga, N.R. Watson. $-2^{\text {nd }}$. ed. - Chichester: Wiley, 2003.

[6] Xiao Yao. The method for designing the third order filter. Proceedings of the 8-th International Conference on Harmonics and Quality of Power. October 14-16, 1998. Athens, Greece, Vol. 1, pp.139-142.

[7] State Standard 13109-97. Power quality. Electromagnetic compatibility of technical equipment. Power quality limits in electrical systems. - M., 1998. (Russia) 